\title{
The Role of Non-Linear Birth Functions on the Dynamics of Malaria Vector Population
}

\author{
Murshed Ahmed Ovi ${ }^{1}$, A.M. Niger ${ }^{2}$ and C.N. Podder ${ }^{* 2}$ \\ ${ }^{1}$ Department of Mathematics, Barishal University, Barishal-8200, Bangladesh \\ ${ }^{2}$ Department of Mathematics, Dhaka University, Dhaka-1000, Bangladesh
}

(Received: 16 August 2018; Accepted: 28 January 2019)

\begin{abstract}
As mosquito vector plays a significant role in malaria dynamics, a deterministic delay differential equation model ${ }^{10}$ for the population dynamics of the malaria vector is rigorously analyzed for the non-delay part subject to a new form of vector birth rate function; the Hassell function. For the Hassell function, the model has a non-trivial equilibrium which is locallyasymptotically stable under certain conditions. It is also shown that the non-trivial equilibrium corresponding to this birth function bifurcates into a limit cycle via a Hopf bifurcation. The Maynard-Smith-Slatkin function is better than the Verhulst-Pearl logistic growth function as the former is associated with increased sustained oscillations 9 . Again this Maynard-Smith-Slatkin function is more preferable than the Hassell function as the prior one is pertained to more sustained oscillations and holds the analyzed properties for the realistic size of the limiting birth rate.
\end{abstract}

Keywords: Malaria, Bifurcation, Limiting birth rate.

\section{Introduction}

Malaria is one of the most acute public health problems in the world. It is a parasitic vector-borne disease and is causing thousands of death in many developing countries, where children and pregnant women are the most vulnerable groups. According to WHO, in 2016, 91 countries and territories had ongoing malaria transmission and an estimated 216 million cases of malaria occurred and caused an estimated 44500 deaths across the globe ${ }^{13}$. People, having limited access to health care and limited ability to afford treatment are disproportionately affected by malaria. Malaria is rampant in a number of countries which cover more than 40 percent of the world's population. The impact of malaria on these countries is not only confined within the health of its people, but also it costs billions of dollars in the treatment and causes loss of productivity each year. Malaria parasite is transmitted from human to human by the Anopheles sp.mosquito due to its human biting habit. The female Anopheles mosquito bites a human being for the sole purpose of harvesting blood that she needs for the development of her eggs. It spreads by infecting female Anopheles mosquitoes and humans successively. At the time of interacting with a human, mosquito can either infect or be infected based on the disease status of both the mosquito and the human. In the process of this interaction, the mosquito may $\operatorname{die}^{3,8}$. Throughout this paper we will consider the malaria transmission caused by mosquito bites. The Anopheles sp. mosquito goes through several distinct stages of development (metamorphosis). This process is temperature dependent and takes up to 2-3 weeks in cold weather ${ }^{3}$. The four species that cause malaria in humans are the protozoan parasites of the genus Plasmodium (Plasmodium vivax, Plasmodium malariae, Plasmodium ovale and Plasmodium falciparum). We will focus on transmission by P.falciparum species only which accounts for the most severe and often potentially lethal forms of malaria ${ }^{7}$. Malaria parasites take two stages to be developed in human one is exoerythrocytic cycle and another is erythrocytic cycle. In exoerythricytic stage thousands of merozoites are produced and the erythrocytic cycle is responsible for maintaining the infection and generating symptoms. Gametocytes (sexual forms of merozoites) are also produced in the erythrocytic stage ${ }^{2,4}$. Fertilization and sexual recombination occurs in the mosquito's gut. Successive erythrocytic cycles result in an increase in parasitaemia until the immune response begins ${ }^{11,12}$. The developing parasites inside a human body destroy red blood cells (RBCs), which may cause death by severe anemia as well as clogging of capillaries that supply the brain, or other vital organs, with blood ${ }^{7}$. Although scientists have been working relentlessly to design a suitable malaria vaccine, no such vaccine is currently available for use in humans. Consequently, anti-malaria strategies are focused on vectorreduction and personal protection. However, in order to choose the most effective vector control strategy, it is necessary to grasp the population dynamics of the malaria vector. In this paper we will consider the model derived and presented in ${ }^{10}$ subject to three forms of birth rate function: the Verhulst-Pearl logistic growth function, the MaynardSmith-Slatkin function and the Hassell function ${ }^{1}$. Our prime goal is to rigorously analyze the theoretical results of the model subject to new form of birth rate function, the Hassell function. Our second objective is to propound a comparative study about the impact of these three birth rate functions on the dynamics of malaria vector to choose the best one.

\section{The Deterministic Model}

At any time $t_{1}$, the density of total adult mosquito population $N_{v}\left(t_{1}\right)$ is divided into three categories $U\left(t_{1}\right)$, $V\left(t_{1}\right)$ and $W\left(t_{1}\right)$ where,

(i) $U\left(t_{1}\right)$, is the collection of fertilized, well-nourished (with blood) and reproducing female vectors.

(ii) $V\left(t_{1}\right)$, is the collection of all previously fertilized female vectors at the breeding site that have just laid down their eggs but are still resting at the breeding

*Author for correspondence. e-mail: cpodder@gmail.com 
site together with all unfertilized female vectors that are not fed with blood and are not questing for blood but swarming at the breeding site.

(iii) $W\left(t_{1}\right)$, is the collection of fertilized but nonreproducing vectors that have left the breeding site for the quest of a blood meal.

Thus $N_{v}\left(t_{1}\right)=U\left(t_{1}\right)+V\left(t_{1}\right)+W\left(t_{1}\right)$

\section{Birth Rate Function}

To choose a birth rate function following definition is to be considered. The per capita birth rate per reproducing vector in class $U$ is denoted by $B(U)$.

Definition 2.1. ${ }^{9}$ A function $B:[0, \infty) \rightarrow \mathbb{R}$ is a suitable birth rate function for the vector if it satisfies the following three conditions.

$\mathrm{A} 1: B(U)>0, \forall U \geq 0$;

A2: $B(U)$ is continuously differentiable with

$B^{\prime}(U)<0, \forall U \geq 0$;

A3: There exists a positive number, called the vectorial basic reproduction number (denoted by $\mathcal{R}_{0}$ ) such that $B(\infty)<\frac{B(0+)}{\mathcal{R}_{0}}<B(0+)=B_{0}$.

\section{Selected Birth Rate Functions}

The functional form of $B(U)$ is chosen based on the fact that, in ecology non-linearity in the dynamics of population of a single species arises due to the competition between the members of the population. Generally when members of the same species compete for a common resource, we notice two types competition namely contest competition and scramble competition ${ }^{6}$. It seems more logical to assume a contest type competition for modeling the dynamics of mosquito population as some of them become successful in drawing blood from human, and some of them die in this process. Consider the following two forms of birth rate functions, which satisfy assumptions A1-A3.

$$
\begin{aligned}
& B 1: B(U)=B_{0}\left(1-\frac{U}{L}\right), U \in[0, L) \\
& B 2: B(U)=\frac{B_{0}}{1+\left(\frac{U}{L}\right)^{n}}, n>0
\end{aligned}
$$

Here $\mathrm{L}$ is considered as the environmental carrying capacity. The functional form $B 1$ is commonly known as the Verhulst-Pearl logistic growth function and the second one i.e. $B 2$ is known as the Maynard-Smith-Slatkin function. These two forms of birth rate function were previously used to describe the dynamics of malaria vector population ${ }^{9,10}$. But there is another function which is also renowned for incorporating both the scramble and contest type competitions ${ }^{1}$ and that is given by

$$
B 3: B(U)=\frac{B_{0}}{\left(1+\frac{U}{L}\right)^{n}}
$$

This function is known as the Hassell function ${ }^{1}$. To the authors knowledge, perhaps this is the first time $B 3$ is being used to model the dynamics of malaria vector population. Now we draw the figure of these three birth rate functions.

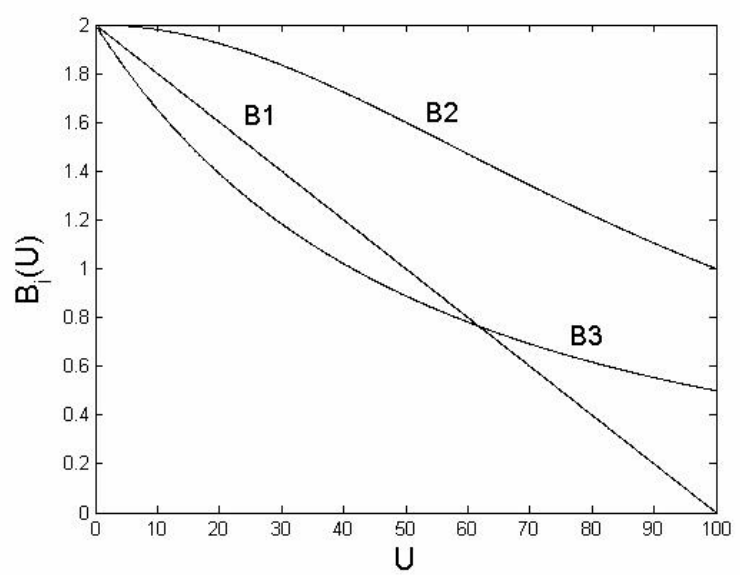

Fig. 1. Graph of birth rate functions $B 1, B 2$ and $B 3$

It follows from the above figure that $B 1$ is linear decreasing function of $U$ while $B 2, B 3$ are non-linear decreasing function of $U$.

\section{The Model}

The deterministic model that will be used is given by the following non-linear system of delay differential equations $^{10}$.

$$
\begin{aligned}
& \frac{d U}{d t_{1}}=\rho \tau H W-(a+\mu) U, \\
& \frac{d V}{d t_{1}}=a B\left(U\left(t_{1}-T\right)\right) U\left(t_{1}-T\right) e^{-\mu_{e} T}+a U-\left(\mu+\frac{b H}{H+K}\right) V \\
& \frac{d W}{d t_{1}}=\left(\frac{b H}{H+K}\right) V-(\mu+\tau H) W
\end{aligned}
$$

With the initial data,

$$
\left(U\left(t_{1}\right), V\left(t_{1}\right), W\left(t_{1}\right)\right)=\left(u_{0}\left(t_{1}\right), v_{0}\left(t_{1}\right), w_{0}\left(t_{1}\right)\right), t_{1} \in[-T, 0]
$$

Where $u_{0}\left(t_{1}\right), v_{0}\left(t_{1}\right)$ and $w_{0}\left(t_{1}\right)$ are continuously differentiable functions on the interval $[-T, 0]$. Here $\tau, H, \rho, \mu, \mu_{e}, a, b$ and $K$ are positive constants and $T>0$ is the delay parameter. The Table 1 describes the model variables and parameters.

Now using the following scaled variables and parameters 
$u(t)=\frac{U}{U_{0}} ; v(t)=\frac{V}{V_{0}} ; w(t)=\frac{W}{W_{0}} ; t=\frac{t_{1}}{T_{0}} ; \alpha=\frac{a U_{0} T_{0}}{V_{0}} ;$

$\rho=\frac{\frac{b H}{H+K}+\mu}{a+\mu} ; \gamma=\frac{\tau H+\mu}{a+\mu} ; \mu_{s}=\frac{\mu_{e}}{a+\mu} ;$

$\mathcal{R}_{0}=\frac{\alpha B_{0}}{\rho-\alpha}, \tau^{*}=\tau H$. Where,

$W_{0}=\frac{(a+\mu)}{p \tau^{*}} U_{0} ; \quad V_{0}=\frac{(a+\mu)\left(\tau^{*}+\mu\right)}{p \tau H \frac{b H}{H+K}} U_{0} ; U_{0}=L ;$ and $\mathrm{T}_{0}=\frac{1}{\mathrm{a}+\mu}$

And also by using $T=0$ (non-delay) the model (4) reduces to

$$
\begin{aligned}
& \frac{d u}{d t}=w(t)-u(t) \\
& \frac{d v}{d t}=\alpha B(L u(t)) u(t)+\alpha u(t)-\rho v(t) \\
& \frac{d w}{d t}=\gamma[v(t)-w(t)]
\end{aligned}
$$

With the initial data

$$
(u(t), v(t), w(t))=\left(u_{0}(t), v_{0}(t), w_{0}(t)\right), t \in[-T, 0]
$$

Applying the above scaling, the birth rate functions now take the forms:

$B(U)=B(L u)=\hat{B}(u)=$
$\left\{\begin{array}{lr}B_{0}(1-u) ; 0<u \leq 1 ; & \text { Type } B 1 \\ \frac{B_{0}}{1+u^{n}} ; & \text { Type } B 2 \\ \frac{B_{0}}{(1+u)^{n}} ; & \text { Type } B 3\end{array}\right.$

For notational convenience $B(u)$ is used in the place of $\widehat{B}(u)$ for each of the birth functions given in (8).

With the help of the scaled variables and parameters and the equation (6) it is easy to show that $\propto<\rho$ and $\propto\left(\tau^{*}\right)=\frac{\alpha_{0} \tau^{*}}{\tau^{*}+\mu}$ where $\alpha_{0}=\left(\frac{\frac{\mathrm{bH}}{\mathrm{H}+\mathrm{K}}+\mu}{\mathrm{a}+\mu}\right)\left(\frac{a}{a+\mu}\right)\left(\frac{\frac{\mathrm{bH}}{\mathrm{H}+\mathrm{K}}}{\frac{\mathrm{bH}}{\mathrm{H}+\mathrm{K}}+\mu}\right) p$.

Also we note that $\gamma\left(\tau^{*}\right)=\frac{\tau^{*}+\mu}{a+\mu} ; \quad Q\left(\tau^{*}\right)=1+\gamma\left(\tau^{*}\right)+$ $\rho$ and $R\left(\tau^{*}\right)=\gamma\left(\tau^{*}\right)+\rho+\rho \gamma\left(\tau^{*}\right)$.

From now on we will introduce $Q, \gamma, R$ instead of referring to the explicit dependence of the parameters on $\tau^{* 9}$.

\section{The Stability of the Trivial Equilibrium}

The model (7) has a trivial equilibrium denoted by $E_{0}=$ $\left(u^{*}, v^{*}, w^{*}\right)=(0,0,0)$, which exists for all parameter values. The following lemma explains the stability state of this trivial equilibrium.

Lemma 3.1. 9 Consider Consider the model (7) with $B:[0, \infty) \rightarrow \mathbb{R}$ satisfying assumptions A1-A3. The trivial equilibrium of the system (7), $E_{0}$ is locally asymptotically stable (LAS) in if $\mathcal{R}_{0}<1$ an unstable if $\mathcal{R}_{0}>1$.
The global asymptotic stability of the trivial equilibrium is established by the following theorem.

Theorem 3.1. ${ }^{9}$ Consider the model (7) with $B:[0, \infty) \rightarrow \mathbb{R}$ satisfying assumptions A1-A3. The trivial equilibrium of the system (7), $E_{0}$ is globally asymptotically stable (GAS) in $\mathbb{R}^{3}$ if $\mathcal{R}_{0}<1$.

The result of the above theorem is numerically illustrated, by simulating the model (7) with appropriate parameter values (so that $\mathcal{R}_{0}<1$ ), corresponding to the Hassell function.
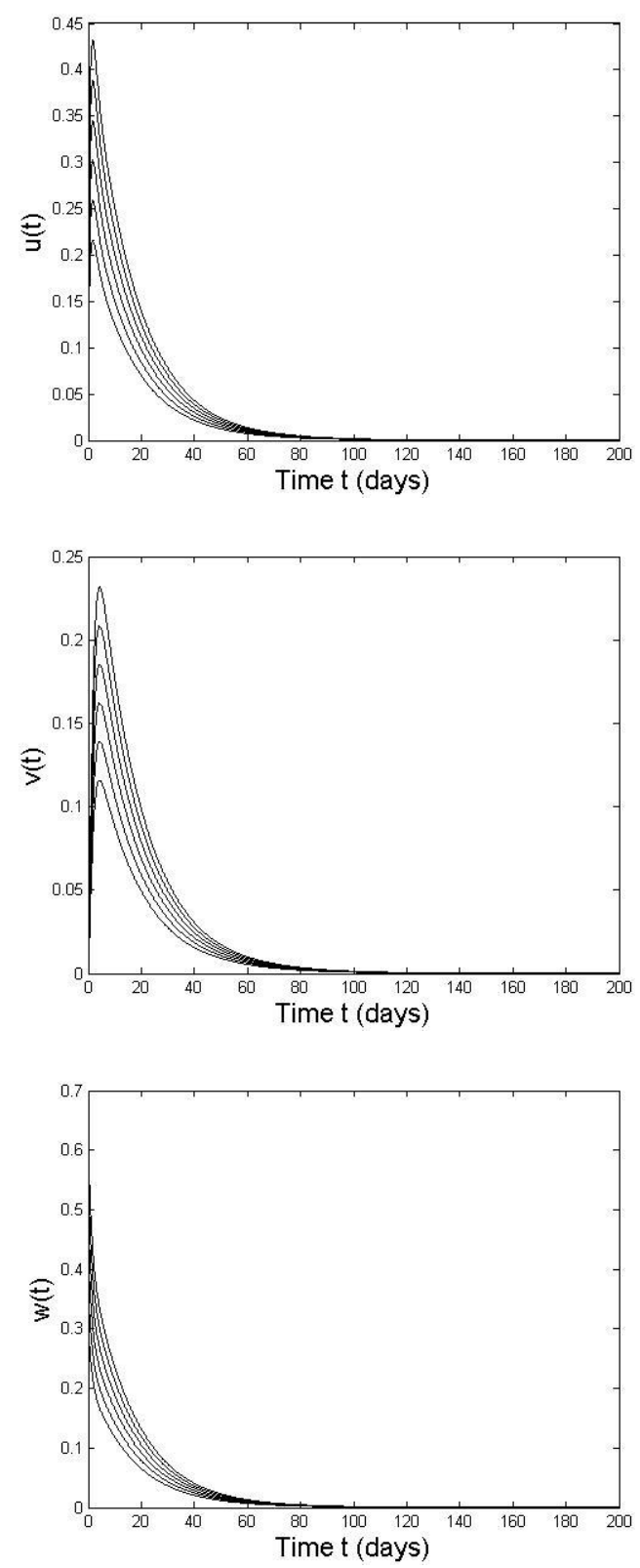

Fig. 2. Simulations of the model (7), using Hassell function B3, showing the state variables converging to the trivial equilibrium for $B_{0}<\frac{\rho-\alpha}{\propto}$. Parameter values used are: $a=1 ; \mu=0.042 ; B_{0}=0.1 ; \alpha=0.3928 ; \rho=$ $0.6161 ; \gamma=.2322 ;$ and $\mathrm{n}=70$ (so that $\left.\mathcal{R}_{0}=.1758<1\right)$. 


\section{Existence and Local Stability of Non-Trivial Equilibrium}

The system (7) has a non-trivial equilibrium point given by $\left(\right.$ see also $^{10}$ )

$E_{1}=\left(u^{* *}, v^{* *}, w^{* *}\right)=(1,1,1) u^{* *}$

Where, $u^{* *}>0$ is obtained as follows. It can be generated by setting the right-hand sides of the equations in (7) equal zero, that is (at non-trivial steady state)

$\propto u^{* *}\left[1+B\left(u^{* *}\right)\right]=\rho v^{* *}=\rho u^{* *}$

So that, $u^{* *}=B^{-1}\left(\frac{B_{0}}{\mathcal{R}_{0}}\right)>0$, where $\mathrm{B}(\mathrm{u})$ is any arbitrary birth function satisfying assumptions A1 and A2, which guarantees the existence of $B^{-1}$. Further, assumption A3, with $B(0+)=B_{0}$, assures the existence of $u^{* *}$ whenever $\mathcal{R}_{0}>1$. Thus the following result holds.

Lemma 3.2. ${ }^{9}$ Consider the model (7) with $B:[0, \infty) \rightarrow \mathbb{R}$ satisfying the assumptions A1-A3. The unique non-trivial equilibrium of (7), given by $E_{1}$ exists whenever $\mathcal{R}_{0}>1$.

For the Hassell function the non-trivial equilibrium becomes,

$E_{1}=\left(u^{* *}, v^{* *}, w^{* *}\right)=\left[(1,1,1)\left(\mathcal{R}_{0}^{\frac{1}{n}}-1\right)\right]$

Now the stability property of this non trivial equilibrium point is given by the following theorem.

Theorem 3.2. ${ }^{9}$ Consider the model (7) with $B:[0, \infty) \rightarrow \mathbb{R}$ satisfying the assumptions A1-A3. The non-trivial equilibrium, $E_{1}$ of the system (7) is LAS whenever $\mathcal{R}_{0}>1$ and $\frac{-A B^{\prime}\left(u^{* *}\right) u^{* *}}{Q R}<1$.

Due to the use of $B 3$ as birth rate function, the local asymptotic stability condition of non-trivial equilibrium $\frac{-A B^{\prime}\left(u^{* *}\right) u^{* *}}{Q R}<1$ implies $B_{0}<\frac{(\gamma n)^{n}(\rho-\alpha)^{n+1}}{\propto[\gamma n(\rho-\alpha)-Q R]^{n}}$ and $\mathcal{R}_{0}<$ $\frac{[\gamma n(\rho-\propto)]^{n}}{[\gamma n(\rho-\propto)-Q R]^{n}}$. The result of the theorem (3.2) is illustrated numerically, by simulating the model (7) with the Hassell function (B3), as depicted in the following Figures 3 and 4

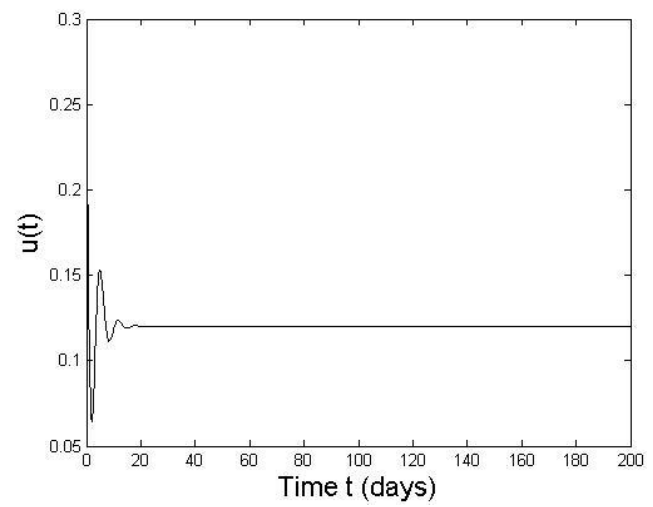

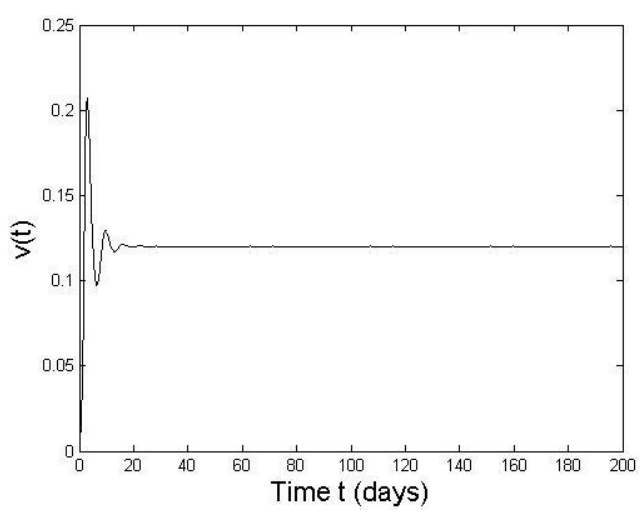

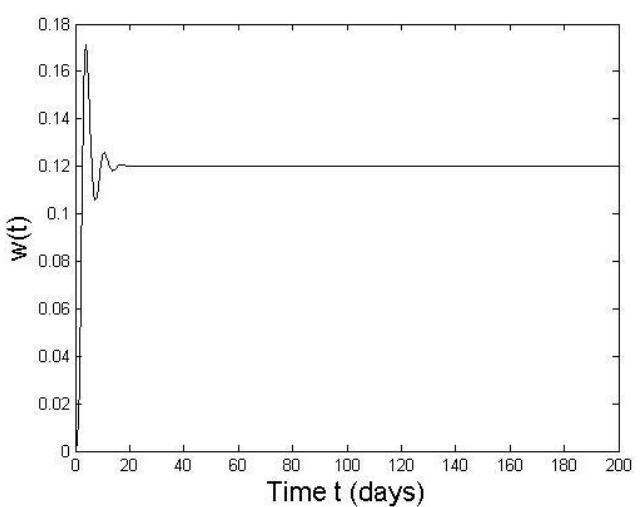

Fig. 3. Simulations of the model (7), using Hassell function B3, showing the time series of the state variables for $\frac{\rho-\alpha}{\alpha}<$ $B_{0}<\frac{(\gamma n)^{n}(\rho-\alpha)^{n+1}}{\propto[\gamma n(\rho-\alpha)-Q R]^{n}}$. Parameter values used are: $a=$ $0.2 ; \mu=0.09 ; B_{0}=300 ; \alpha=0.0984 ; \rho=$ $0.6552 ; \gamma=1$; and $\mathrm{n}=35$ (so that $1<\mathcal{R}_{0}=53.0231<$ $\left.\frac{[\gamma n(\rho-\alpha)]^{n}}{[\gamma n(\rho-\alpha)-Q R]^{n}}=5.57 \times 10^{5}\right)$.

So these analyses reveal that the model (7) subject to $B 3$ exhibits the same equilibrium dynamics as the birth rate functions B1 and B2 ${ }^{9}$. That is, even for B3 the model shows it's convergence to $E_{0}$ for $\mathcal{R}_{0}<1$ and to $E_{1}$ for $\mathcal{R}_{0}>1$ and $\frac{-A B^{\prime}\left(u^{* *}\right) u^{* *}}{Q R}<1$. 

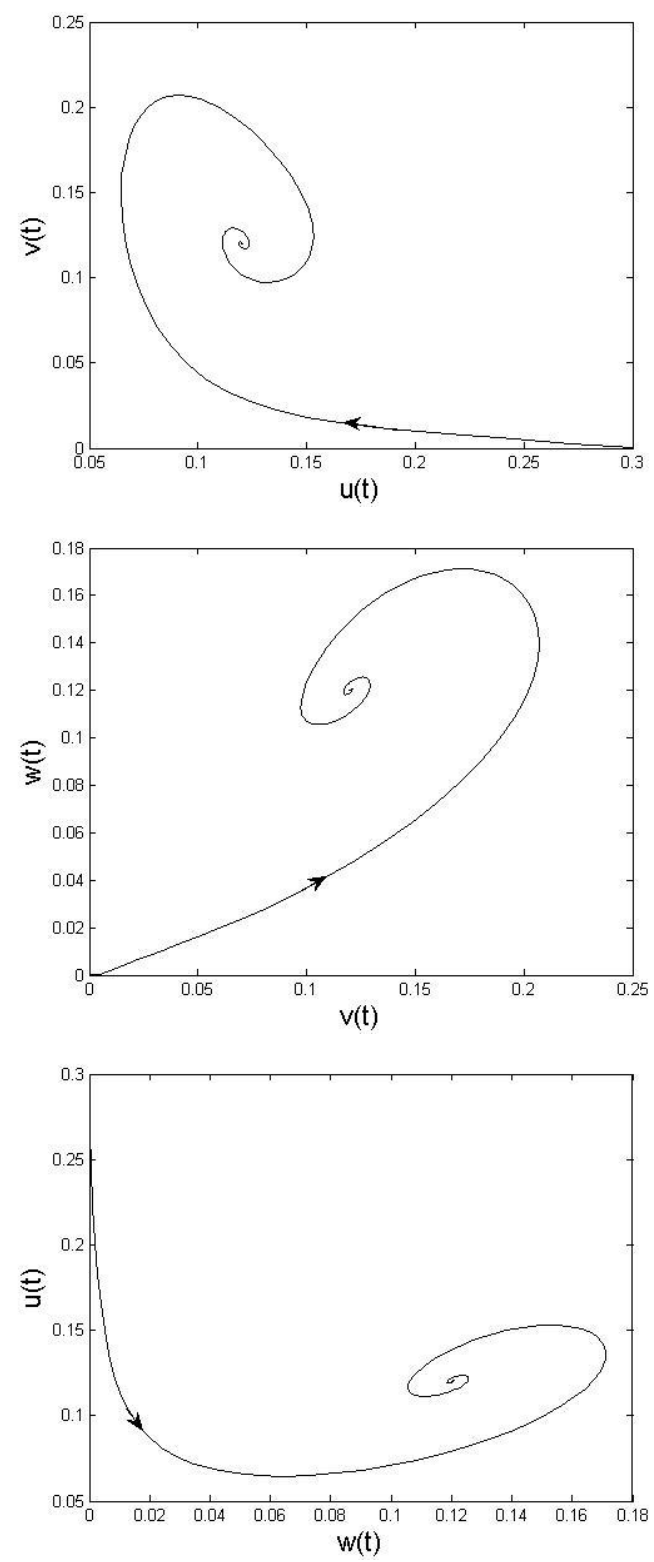

Fig. 4. Simulations of the model (7), using Hassell function B3, showing the time series of the state variables for $\frac{\rho-\alpha}{\alpha}<$ $B_{0}<\frac{(\gamma n)^{n}(\rho-\alpha)^{n+1}}{\alpha[\gamma n(\rho-\alpha)-Q R]^{n}}$. Parameter values used are: $a=$ $0.2 ; \mu=0.09 ; B_{0}=300 ; \alpha=0.0984 ; \rho=$ $0.6552 ; \gamma=1$; and $\mathrm{n}=35$ (so that $1<\mathcal{R}_{0}=$ $\left.53.0231<\frac{[\gamma n(\rho-\alpha)]^{n}}{[\gamma n(\rho-\alpha)-Q R]^{n}}=5.57 \times 10^{5}\right)$.

\section{Existence of Hopf Bifurcation}

The consideration of the inequality $\frac{-A B^{\prime}\left(u^{* *}\right) u^{* *}}{Q R}<1$ as the equality i.e. $\frac{-A B^{\prime}\left(u^{* *}\right) u^{* *}}{Q R}=1$ is required to show that the system may undergo a Hopf bifurcation at a point in the parameter space ${ }^{9}$.
Lemma 4.1. For the birth rate functions B3 and for every parameter grouping, there exists a positive number $\gamma^{*}$ such that,

$\frac{-\propto \gamma^{*} u^{* *} B^{\prime}\left(u^{* *}\right)}{Q\left(\gamma^{*}\right) R\left(\gamma^{*}\right)}=1$

Proof: Consider the bifurcation condition $\frac{-\propto \gamma^{*} u^{* *} B^{\prime}\left(u^{* *}\right)}{Q\left(\gamma^{*}\right) R\left(\gamma^{*}\right)}=1$. Thus $\gamma^{*}$ is determined by the fixed point of the map

$f: y \mapsto-\frac{(1+\rho+y)[\rho+(1+\rho) y]}{\propto u^{* *} B^{\prime}\left(u^{* *}\right)}$ such that $y \epsilon(0, \infty)$. In another way, the required value of $\gamma^{*}$ at the bifurcation point is given by a positive real solution of $y$ of the linear equation

$y+\frac{(1+\rho+y)[\rho+(1+\rho) y]}{\propto u^{* *} B^{\prime}\left(u^{* *}\right)}=0$

After simplification (11) implies

$a_{1} y^{2}+b_{1} y+c_{1}=0$

Where $a_{1}=(1+\rho) ; b_{1}=\left[(1+\rho)^{2}+\rho+\propto u^{* *} B^{\prime}\left(u^{* *}\right)\right]$; $c_{1}=\rho(1+\rho)$.

Clearly, the constants $a_{1}$ and $c_{1}$ are positive. Thus the quadratic equation (12) will have two positive roots if $b_{1}>0$ and $b_{1}{ }^{2}-4 a_{1} c_{1} \geq 0$.

The positive solutions of the quadratic equation (12) are given by

$\gamma_{1}{ }^{*}=y_{1}=\frac{-b_{1}-\sqrt{b_{1}{ }^{2}-4 a_{1} c_{1}}}{2 a_{1}}$
$\gamma_{2}{ }^{*}=y_{2}=\frac{-b_{1}+\sqrt{b_{1}{ }^{2}-4 a_{1} c_{1}}}{2 a_{1}}$

In particular, $\gamma_{1}{ }^{*}$ takes the following form for the birth rate function $B 3$

$\gamma_{1}{ }^{*}=\frac{1}{2(1+\rho)}\left\{-\left[(1+\rho)^{2}+\rho-\propto n B_{0}\left(\frac{1}{\mathcal{R}_{0}}-\frac{1}{\mathcal{R}_{0} \frac{n+1}{n}}\right)\right]-\right.$

$\left.\sqrt{\left[(1+\rho)^{2}+\rho-\propto n B_{0}\left(\frac{1}{\mathcal{R}_{0}}-\frac{1}{\mathcal{R}_{0} \frac{n+1}{n}}\right)\right]^{2}-4 \rho(1+\rho)^{2}}\right\}$

Theorem 4.1. Consider the system (7) with $B(u)=\frac{B_{0}}{(1+u)^{n}}$ and $\gamma_{1}{ }^{*}$ as defined in (14). The system undergoes a Hopf bifurcation at $\gamma_{1}{ }^{*}$.

Proof: Consider $\gamma$ as the bifurcation parameter and $\gamma_{1}{ }^{*}$ as defined in (14). It can be easily shown that if $\lambda$ is an eigenvalue of the characteristic polynomial at the nontrivial equilibrium then $\lambda$ satisfies

$\left(\right.$ since $\left.Q R=\gamma n(\rho-\propto)\left(1-\frac{1}{\mathcal{R}_{0}^{\frac{1}{n}}}\right)\right)$ 
$\lambda^{3}+Q \lambda^{2}+R \lambda+\gamma n(\rho-\propto)\left(1-\frac{1}{\mathcal{R}_{0} \frac{1}{n}}\right)=0$

At $\gamma=\gamma_{1}{ }^{*}$, the cubic equation (15) has a pair of purely imaginary roots, given by $\lambda= \pm i \sqrt{R}$. Substitution of $\lambda= \pm i \omega$ (with $\omega>0$ ), in (15) provide a real part and an imaginary part and after some algebraic manipulation the following is produced.

$$
\begin{aligned}
& \frac{\partial \operatorname{Re}(\lambda)}{\partial \gamma} \\
& =\frac{1}{2}\left\{-\left(1+\rho+\rho^{2}\right)\right. \\
& -\sqrt{\left[(1+\rho)^{2}+\rho-\propto n B_{0}\left(\frac{1}{\mathcal{R}_{0}}-\frac{1}{\mathcal{R}_{0} \frac{n+1}{n}}\right)\right]^{2}-4 \rho(1+\rho)^{2}} \\
& \left.-n(\rho-\alpha)\left(1-\frac{1}{\mathcal{R}_{0} \frac{1}{n}}\right)\right\}<0\left(\text { since } b_{1}{ }^{2}-4 a_{1} c_{1}>0\right)
\end{aligned}
$$

Thus Hopf bifurcation occurs at $\gamma=\gamma_{1}{ }^{*}$ given by (14).

The result of the theorem (4.1) is illustrated numerically by simulating the model (7) with $B 3$, as shown in the following figures.
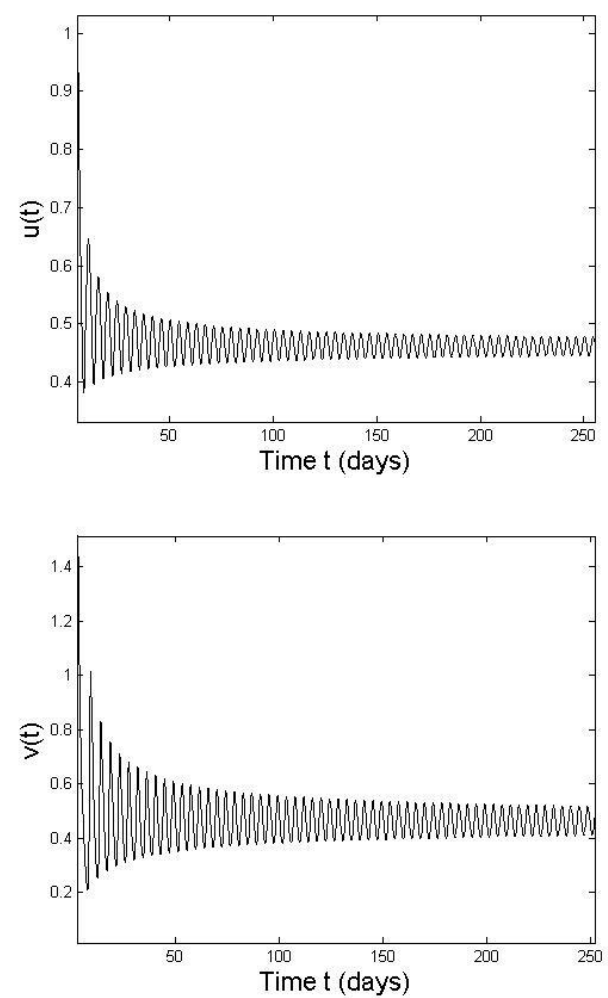

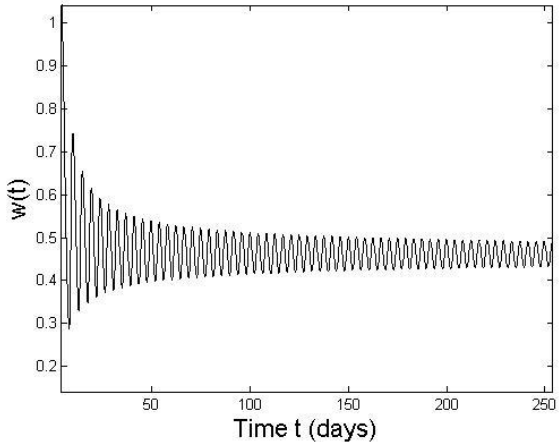

Fig. 5. Simulations of the model (7), using Hassell function B3, showing the time series of the state variables for $B_{0}>$ $\frac{(\gamma n)^{n}(\rho-\alpha)^{n+1}}{\propto[\gamma n(\rho-\alpha)-Q R]^{n}}$. Parameter values used are: $a=0.2 ; \mu=$ $0.09 ; B_{0}=3.2 \times 10^{6} ; \alpha=0.0984 ; \rho=0.6552 ; \gamma=$ 1 ; and $\mathrm{n}=35\left(\right.$ so that $\mathcal{R}_{0}=5.68 \times 10^{5}>$ $\left.\frac{[\gamma n(\rho-\propto)]^{n}}{[\gamma n(\rho-\propto)-Q R]^{n}}=5.57 \times 10^{5}\right)$.
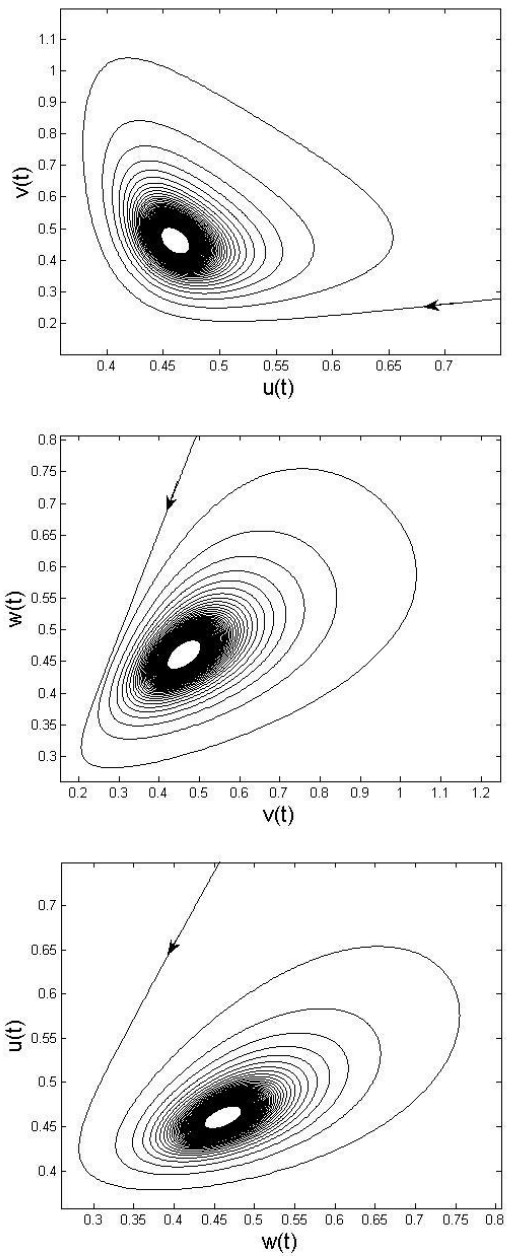

Fig. 6. Simulations of the model (7), using Hassell function B3, showing the time series of the state variables for $B_{0}>$ $\frac{(\gamma n)^{n}(\rho-\alpha)^{n+1}}{\propto[\gamma n(\rho-\alpha)-Q R]^{n}}$. Parameter values used are: $a=0.2 ; \mu=$ $0.09 ; B_{0}=3.2 \times 10^{6} ; \alpha=0.0984 ; \rho=0.6552 ; \gamma=$ 1 ; and $\mathrm{n}=35\left(\right.$ so that $\mathcal{R}_{0}=5.68 \times 10^{5}>\frac{[\gamma n(\rho-\alpha)]^{n}}{[\gamma n(\rho-\alpha)-Q R]^{n}}=$ $\left.5.57 \times 10^{5}\right)$. 


\section{Simulation Analysis Corresponding to B3}

During the generation of the figure (3) and (4) to show the local asymptotic stability of the non-trivial equilibrium $E_{1}$ the limiting birth rate $B_{0}=300$ has been used. The figure (3) is suggesting us that the Hassell function i.e. B3 is not associated with the increased sustained oscillations but these oscillations becomes more sustained if the value of $B_{0}$ is increased which is not realistic $\left(B_{0}\right.$ is the limiting birth rate when the population size is small). Again to show the existence of Hopf bifurcation (figure (5) and (6)) a huge limiting birth rate i.e. $B_{0}=3200000$ has been used. Although it has been tried to show the existence by using different set of parameter values and much lesser values for $B_{0}$ but failed to meet the expectation.

\section{Conclusion}

The Maynard-Smith-Slatkin birth function is more suitable than the Verhulst-Pearl logistic growth function for modeling the dynamics of malaria vector as the previous one is associated with increased sustained oscillations which is desired ecological feature, since it assures the existence of the vector in the ecosystem ${ }^{9}$.

Again the Maynard-Smith-Slatkin function is more preferable than the Hassell function as the prior one is related with increased sustained oscillations and holds the analyzed properties for the realistic size of the limiting birth rate.

Hence we can conclude that the Maynard-Smith-Slatkin function is one of the best choices as a birth rate function for modelling the dynamics of malaria vector population.

Table 1. Description of variables and parameters of the model (4).

\begin{tabular}{|c|c|}
\hline $\begin{array}{l}\text { Variables and } \\
\text { Parameters }\end{array}$ & Descriptions \\
\hline$\rho$ & $\begin{array}{l}\text { Probability of successfully taking a } \\
\text { blood meal. }\end{array}$ \\
\hline$\mu$ & Natural death rate of adult vectors. \\
\hline$\mu_{e}$ & $\begin{array}{l}\text { Natural death rate of vectors in } \\
\text { earlier development stages. }\end{array}$ \\
\hline$\tau$ & $\begin{array}{l}\text { Contact rate between vectors of } \\
\text { type } \mathrm{W} \text { and humans. }\end{array}$ \\
\hline$H$ & $\begin{array}{l}\text { Population density of humans at the } \\
\text { human habitat sites. }\end{array}$ \\
\hline$B$ & $\begin{array}{l}\text { Rate at which fertilized and fed } \\
\text { vectors lay eggs. }\end{array}$ \\
\hline$a$ & $\begin{array}{l}\text { Rate of return of vectors to the } \\
\text { vector breeding site }\end{array}$ \\
\hline$b$ & $\begin{array}{l}\text { Rate at which } V \text { type vectors visits } \\
\text { human habitat sites. }\end{array}$ \\
\hline$K$ & Alternative food resources. \\
\hline
\end{tabular}

\section{References}

1. Brännström, Ā. and Sumpter, J. T. David, 2005. The role of competition and clustering in population dynamics. Proceedings of the Royal Society of London B: Biological Sciences, 272(1576), 2065-2072.

2. Diebner, H. H., M. Eichner, L. Molineaux, W. E. Collins, G. M. Jeffery, \& K. Dietz, 2000. Modelling the transition of asexual blood stages of Plasmodium falciparum to gametocytes. Journal of Theoretical Biology, 202(2), 113-127.

3. Giles, H. M. and D. A. Warrel, 1993. Bruce-Chwatt's Essential Malariology, 3rd Edition. Heinemann Medical Books, Portsmouth, NH.

4. Hoshen, M. B., R. Heinrich, W. D. Stein, \& H. Ginsburg, 2000. Mathematical modelling of the within-host dynamics of Plasmodium falciparum. Parasitology, 121(3), 227-235.

5. https://www.nothingbutnets.net/new/saving-lives/how-doesmalaria-impact-communities.html (Accessed on May, 2017).

6. Jang, S. J., 2005. Contest and scramble competition with a dynamic resource. Nonlinear Analysis: Theory, Methods \& Applications, 63(5), 109-118.

7. Malaria Site. http://malariasite.com (Accessed April, 2017).

8. McCall, P. J., \& D. W. Kelly, 2002. Learning and memory in disease vectors. Trends in parasitology, 18(10), 429-433.

9. Ngwa, G. A., A. M. Niger, \& A. B. Gumel, 2010. Mathematical assessment of the role of non-linear birth and maturation delay in the population dynamics of the malaria vector. Applied Mathematics and Computation, 217(7), 32863313.

10. Ngwa, G. A. 2006. On the population dynamics of the malaria vector. Bulletin of Mathematical Biology, 68(8), 2161-2189.

11. Niger, A. M., \& A. B. Gumel, 2011. Immune response and imperfect vaccine in malaria dynamics. Mathematical Population Studies, 18(2), 55-86.

12. Niger, A. M., \& A. B. Gumel, 2008. Mathematical analysis of the role of repeated exposure on malaria transmission dynamics. Differential Equations and Dynamical Systems, 16(3), 251-287.

13. World Health Organization, 2017. World Malaria Report.http://www.who.int/malaria/media/world-malariaday-2017/en/. (Accessed on August 2018). 
